\title{
Erratum to: Short and long access to cocaine self-administration activates tyrosine phosphatase STEP and attenuates GluN expression but differentially regulates GluA expression in the prefrontal cortex
}

\author{
Wei-Lun Sun • Agnieszka Zelek-Molik • \\ Jacqueline F. McGinty
}

Published online: 5 June 2013

(C) Springer-Verlag Berlin Heidelberg 2013

\section{Erratum to: Psychopharmacology}

\section{DOI 10.1007/s00213-013-3118-5}

The original version of this article inadvertently contained a mistake.

An incorrect version of Figure 4 was published. The correct figure appears below.
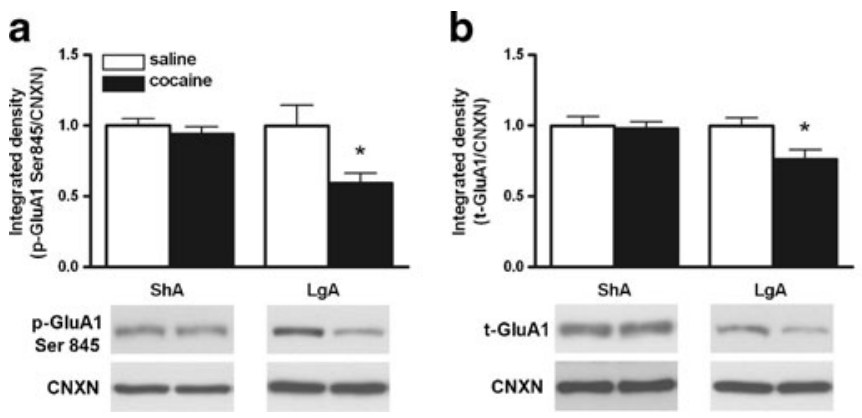

Fig. 4 Effects of ShA and LgA cocaine self-administration on protein levels of p-GluA1 (a), t-GluA1 (b), p-GluA1 Ser845/t-GluA1 ratio (c), and t-GluA2 (d) in the dmPFC. Lower panels represent typical Western
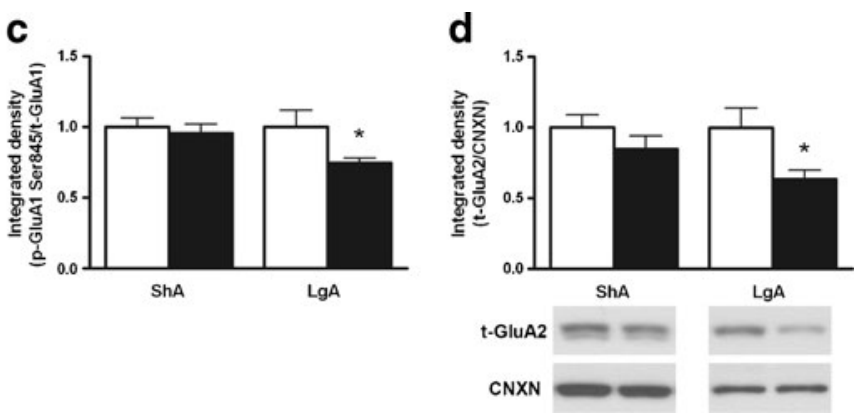

blot images for each protein. Significant differences from the respective yoked saline controls are indicated $\left({ }^{*} p<0.05 ; N=6-9\right.$ per group)

The online version of the original article can be found at http://dx.doi.org/ 10.1007/s00213-013-3118-5.

W.-L. Sun · A. Zelek-Molik • J. F. McGinty $(\square)$

Department of Neurosciences, Medical University of South

Carolina, 173 Ashley Ave MSC 510,

Charleston SC 29425, USA

e-mail: mcginty@musc.edu 